\title{
On a Transition Phase in Single Well Tests Data
}

\author{
Jiří Gregor ${ }^{1} \&$ František Pastuszek ${ }^{2}$
}

\author{
${ }^{1}$ Dept.of Mathematics, Faculty of Electrical Eng., Czech Technical University, Prague \\ ${ }^{2}$ Institute of Hydrogeology, Engineering Geology and Applied Geophysics, Charles University, Prague
}

Correspondence: Jiří Gregor, Dept.of Mathematics, Faculty of Electrical Eng., Czech Technical University, Prague. Email: gregorj4@gmail.com

Received: October 30, 2018

Accepted: November 15, 2018

Online Published: November 30, 2018

doi:10.5539/enrr.v18n4p46

URL: https://doi.org/10.5539/enrr.v8n4p46

\begin{abstract}
In processing data of pumping tests of single wells at constant pumping rate the drawdown during the first fraction of minutes is modeled by a linear function of time as the well-bore storage. After a few minutes of pumping the drawdown is considered to be described by a solution of the Theis differential equation. The time span between these two "regimes" is here named transition phase of the test. Models of this phase are suggested. These models were tested on a number of data sets, some results are shown in the closing part of this communication. It turns out that data in the transition phase contain significant information on the vicinity of the bore.
\end{abstract}

Keywords: hydraulic losses, single well tests, skin factor

Boring a well results in significant destruction of homogenity of the aquifer in the vicinity of the bore. Data obtained on drawdown reflect these and other changes (e.g.clogging) which are commonly summarized as hydraulic losses. Their quantification was suggested e.g. in (Fenske,1984), (Hantush \& Jacob,1955), (Ramey,1970) in various ways. Since the difference between the pumping rate and inflow rate linearly depends on the derivative of drawdown (Gregor and Pastuszek, 2018) a simple mathematical model can be suggested. It is an alternative approach to the skin effect (van Everdingen, 1953) or skin factor (Houze, Viterat \& Fjaere, 2008) and yields an quantified view into processes in the neighborhood of the bore.

\section{Models}

The models should be functions of time, continuous and increasing for $t \in\left(t_{1}, t_{2}\right.$, ) where $t_{1}$ and $t_{2}$ stand for the end of well bore storage and for the begin of the "normal" flow, respectively. They have to give a true picture of drawdown. Denote these functions as $s_{i}$ and start with $s_{1}$.

1. After $t_{1}$ water is pouring into the bore at a rate $Q-\pi r^{2} s_{1}^{\prime}(t)$ as shown in (Gregor and Pastuszek, 2018). On the other hand, this influx rate is proportional to the area through which it occurs. This area can be evaluated as $2 \pi r s_{1}[t]$, where $r$ is the radius of the bore. Equating these quantities a simple first order linear differential equation is obtained:

$$
Q-\pi r^{2} s_{1}^{\prime}(t)=2 k \pi r s_{1}[t]
$$

where $k$ is the coefficient of proportionality of dimension $[\mathrm{m} / \mathrm{sec}]$. To assure continuity of the model from the start of pumping till $t_{2}$ the initial condition should be $s_{1}\left[t_{1}\right]=w\left[t_{1}\right]$, where $w$ denotes the linear model of well bore storage , e.g. as $w[t]=a+b t$. The model of the transition phase follows as a solution $s_{1}[t]$ of the above differential equation.

$$
s_{1}[t]=z+\left(w\left[t_{1}\right]-z\right) \exp \left[-\frac{2 k\left(t-t_{1}\right)}{r}\right]
$$

where $z=\frac{Q}{2 \pi r k}$. Here all constants are known except for $k$, the permeability, which can be easily estimated by least square method from measured data. Note that $\lim _{\rightarrow \infty}=z$. This value can be interpreted as a steady state in a hypothetical homogeneous aquifer of properties identical to those in the vicinity of the well, i.e. it corresponds to all losses in well-bore neighborhood.

Note also that considering the pumped well as a linear time-invariant black-box of first order we arrive at the equation of a chemical reactor, where the corresponding first order linear differential equation with above initial conditions has the solution

$$
s_{2}[t]=K+\left(w\left[t_{1}\right]-K\right) \exp \left[\alpha\left(t_{1}-t\right)\right] .
$$


$K \alpha$ represents the flowing rate of the reactor. $K=Q / \hat{T}$, where $Q$ is the pumping rate and $\hat{T}$ equals to specific capacity. The time constant $\alpha$ for a reactor often corresponds to the ratio $Q / V$, where $Q$ and $V$ equals to the flow and volume of the reactor. Here $V$ stands for the volume of water in the clogging zone and therefore the ratio of this value and the volume of the clogging zone can be taken as an estimate of effective porosity of the clogging zone. In above explained terms and symbols this estimate is expressed as

$$
\frac{Q}{2 \pi s\left[t_{1}\right]\left(\delta^{2}-r^{2}\right)} .
$$

There is a one-to-one correspondence between parameters in (2) and (3),

$$
K=\frac{Q}{2 \pi r k} \quad \alpha=\frac{2 k}{r}
$$

i.e. they define one model. Nevertheless, in numerical handling with data they could differ. Comparison may serve as a check of correctness in parameter estimates.

Semi-logarithmic plot of data contains a part easily modeled by a straight line, which suggests the exponential growth as described above but its slope has a rather unclear physical meaning. The above equations (2) and (3) yield it.

2. A published model of the transition phase due to Hantush is described in (Batu,1998) which is included here for comparison. It is based on the concept of a "mirror well" or imaginary well to which water is flowing at a rate of pumping, i.e. assuming the existence of an impenetrable wall at a certain distance from the bore axis.

The aquifer is here supposed to remain unchanged for all values of $t$, i.e. with all the losses remaining constant. This Hantush model reads as follows.

$$
s_{3}[r, t]=\frac{Q}{4 \pi T}\left(\Gamma\left[0, \frac{r^{2} \sigma}{4 T t}\right]-\Gamma\left[0, \frac{(2 \delta-r)^{2} \sigma}{4 T t}\right]\right),
$$

where $2 \delta$ stands for the distance of the mirror well from the bore axis and $\delta$ nay serve as an estimate of the width of the clogging zone. $T=T H, \sigma=\sigma H$ can be considered as some mean values of transmisivity and storativity in the neighborhood of the well, which are often estimated using data of an observation well. Results of application of this model after evaluation of its parameters $T H, \sigma H, \delta$ are shown below.

The described models are physically motivated, offer important information on the close vicinity of the bore such as its hydraulic conductivity, "losses" caused by non-homogenities, characteristic of the clogging zone, they enable to quantify the so called "skin factor" and last but not least they offer a way to create a continuous model of all data obtained in the test. Their properties, comparisons and actual results of their use follow.

\section{Applications and discussion}

Application of suggested models should start with estimates of their parameters from given data and a check of their accuracy. Some examples using mean square technics follow.

As a first one results from a well at Vlastislav in North Bohemia, Czech Republic, are presented. Data denoted as m01b and m01a were obtained before and after regeneration of the bore, respectively ( Data in .xlsx formate are available upon request via e-mail). Results are shown in Fig.1. It contains beside the suggested model also the Hantush model (black) for comparison. The parameters of the bore, results of parameter estimates and some derived quantities (in standard units) are summarized in Table 1 .

Table 1. Parameters of models for the well m01 before and after regeneration

\begin{tabular}{cccccccc}
\hline & $Q$ & $r$ & $k$ & $z(\approx K)$ & timespan & $\lambda$ & $\delta$ \\
\hline m01 b & 0.00295 & 0.1625 & 0.00155 & 1.8604 & $(20,170)$ & 1.89 & 0.458 \\
m01 a & 0.00148 & 0.1625 & 0.00245 & 0.59165 & $(8,110)$ & 0.683 & 0.4217 \\
\hline
\end{tabular}

Relative errors of the models during the whole time span were considerably less than $0.01 \%$. A comparison of the two data sets for the bore $\mathrm{m} 01$ effectively demonstrates the effect of regeneration of the bore: the time span of transition phase became shorter as well as the time of well bore storage. Permeability has increased, the clogging zone diminished and the hypothetical steady state (assuming that conditions of the transition phase remain unchanged) related to pumping rate 

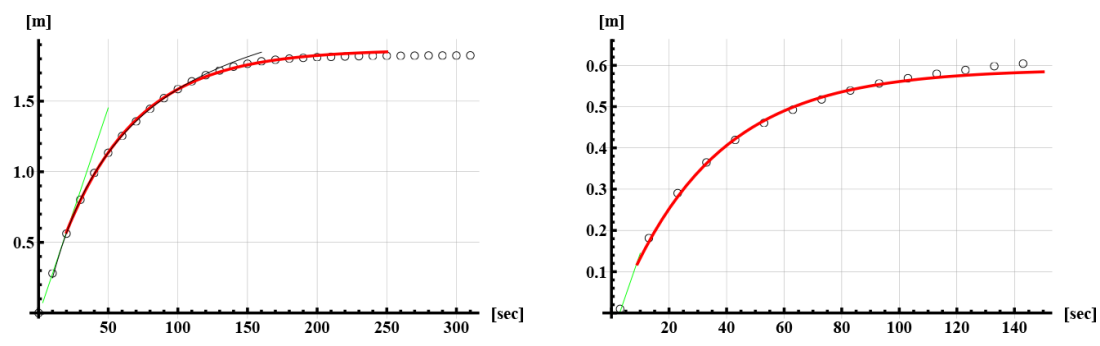

Figure 1: Data and models of transition phase

was reduced by $1 / 3$. All these effects are quantified and as such can be compared to other data sets. Last but not least the model give a good estimate of the time when unhindered flow can be described by a suitable solution of the Theis equation to find reliable estimates of transmissivity a storativity of the aquifer.

Such solution of the Theis equation is a function of two variables, time and distance from the axis of the bore. To assess (or predict) drawdown due to pumping at arbitrary distance, e.g. at an observation well it has to taken into account that data of the drawdown differ from those outside of the well by the so called "jump on the skin". The above model enable to asses this difference, i.e. to quantify this jump. To this end it is sufficient to solve equation (1) with zero initial conditions so as to obtain a model "predicting" the transition phase at the outward side of the well. Details of such step are out of the scope of this short communication as they demand to discuss the appropriate solution of the Theis equation. Authors will summarize corresponding results elsewhere.

\section{References}

Batu, V. (1998). Aquifer hydraulics: a comprehensive guide to hydrogeologic data analysis (Vol. 1). New York: John Wiley Sons.

Fenske B. A. (1984). Unsteady drawdown in the presence of a linear discontinuity. Groundwater Hydraulics, 9, 109-145

Gregor, J., \& Pastuszek, F. (2018). On the derivative of drawdown in single well test data. Water Resourcees Management, 32(13), 4155-4158.

Hantush, M. S., \& Jacob, C. E. (1955). Non-steady radial flow in an infinite leaky aquifer. Trans. Amer. Geophys. Un., $36,995-100$.

Houze, O., Viterat, D., \& Fjaere O. S. (2008). Dynamic flow analysis. Kappa.

Ramey Jr, H. J. (1970). Short-time well test data interpretation in the presence of skin effect and wellbore storage. Journal of Petroleum Technology, 22(01), 97-104.

van Everdingen, A. F. (1953). The skin effect and its influence on the productive capacity of a well. Trans. AIME, 198, 171-176.

\section{Copyrights}

Copyright for this article is retained by the author(s), with first publication rights granted to the journal.

This is an open-access article distributed under the terms and conditions of the Creative Commons Attribution license (http://creativecommons.org/licenses/by/4.0/). 